\title{
Retrospective Study Of The Management Of
}

\section{Suspected Febrile Neutropenia In A Single-Centre Metropolitan Western Sydney Hospital}

This article was published in the following Dove Press journal: Clinical Audit

\author{
Samuel J Doyle' \\ Joanne H Mackson (D) ${ }^{\prime}$ \\ Mark D Salter ${ }^{1,2}$ \\ 'Emergency Department, Nepean \\ Hospital NSW, Kingswood, NSW, \\ Australia; ${ }^{2}$ School of Medicine, University \\ of Sydney, Sydney, NSW, Australia
}

Correspondence: Mark D Salter

Emergency Department, Nepean Hospital

NSW, Kingswood, NSW, Australia

Email mark.salter@health.nsw.gov.au
Objective: Febrile neutropenia is an oncological emergency, associated with considerable morbidity and mortality. The mainstay of initial treatment is broad-spectrum intravenous antibiotics. We aim to determine our level of performance primarily by determining the time to initial antibiotic administration amongst other secondary outcomes. We propose that this may assist in providing an improvement in service provision and the clinical outcomes of patients from updated and more site-centred recommendations.

Methods: A retrospective cohort study was conducted at the Nepean Hospital Emergency Department by two independent researchers from the hospital electronic and paper medical records. The primary outcome for the study was the time to initial antibiotics. Secondary outcomes included time to initial litre of intravenous fluids, time to antipyretics, number of positive culture or imaging results and the first-line antibiotic choice.

Results: A total of 244 patients were included. 127 patients $(52.05 \%)$ received either Piperacillin-Tazobactam or cefepime, with or without gentamicin, which is congruent with the local clinical pathway. Mean time to administration of antibiotics for the cohort was 127 mins (82; 49.5-149 mins). Initial Intravenous fluid boluses were given to 177 patients (72.54\%), with a mean time 166 mins (117; 64-196 mins) whilst 161 patients $(65.98 \%)$ received antipyretics within 160 mins (90; 53-208 mins).

Conclusion: The results show a delay in treatment and variation in the selection of initial antibiotics at our centre. The results should lead to changes in staff education on the existence of guidelines, their recommendations and how the processes for the initiation of therapy can be improved at our centre. This may encourage other services to ascertain their performance and encourage them to identify obstacles to efficient and effective care to improve patient outcomes.

Keywords: febrile neutropenia, antibiotics, sepsis

\section{Introduction}

Febrile neutropenia is a medical emergency and well-recognised complication of chemotherapy, associated with considerable morbidity and mortalty. ${ }^{1}$ In up to $30 \%$ of cases there is associated bacteremia and a risk of developing sepsis and further complications. . $^{2,3}$

Febrile neutropenia affects between $10 \%$ and $50 \%$ of patients with solid tumour and $80 \%$ of patients with haematological malignancies and can occur in anyone receiving chemotherapy ${ }^{4}$ The mainstay of initial treatment is empirical broad-spectrum intravenous (IV) antibiotics and prompt administration is fundamental to patient survival and 
reduced length of hospital admissions. ${ }^{5-8}$ Previous studies have estimated that greater than $50 \%$ of febrile neutropenic patients will develop sepsis. Therefore, treatment should be commenced before initial investigation results are available to minimise delays. ${ }^{9-11}$

There are factors that may prevent patients from receiving antibiotics within the recommended timeframe of 1 hour. ${ }^{3}$ Patients often present to centres different from the hospital-administering chemotherapy or present out of hours, resulting in the risk of neutropenia not being identified. ${ }^{4}$ Emergency department waiting times, staffing levels, resource allocation and financial constraints as well as physician variability with knowledge of treatment guidelines and what requires in-patient care can also affect the time to antibiotic administration. ${ }^{12,13}$

Nepean Hospital has a busy haematology and oncology service, with a flow on effect being potential febrile neutropenic patients presenting to the Emergency Department (ED). The ED febrile neutropenic guideline suggests that all patients presenting as "hot and high risk" (at risk for febrile neutropenia) should receive initial investigations including blood cultures, IV access and IV antibiotics within $1 \mathrm{hr}$ of presentation. The aim of this clinical audit is to determine whether these patients are being treated appropriately as per the febrile neutropenia guideline.

\section{Methods}

\section{Study Design}

The study was conducted at Nepean Hospital, a 520-bed metropolitan Hospital in Western Sydney. The study was designed as a retrospective cohort chart review of patients who were triaged as a category 2 "hot and high risk", using the Manchester Triage System ${ }^{14}$ from January 2017-December 2017. In order to fulfill the triage criteria for inclusion into the "hot and high risk" category, the patient had to present with pyrexia at triage observation, or give a history of an objective fever at home and received chemotherapy within 1 month of presentation. ${ }^{15}$ Patients' inclusion criteria also included them being $\geq 18$ years of age as there is no paediatric oncology department at Nepean Hospital. The audit aimed to look at the initial treatment of patients who are "at risk" of febrile neutropenia by triage classification. Information regarding the patient's current chemotherapy regimen was not available at initial presentation due to differing clinical systems being used by the local cancer care departments.
The study was approved by the Nepean-Blue Mountains Local Health District Human Research Ethics Committee as a retrospective clinical audit and was exempt from the requirement for informed patient consent. Neutropenia is typically characterized by international standards by a reduction in neutrophils below normal counts, usually occurring within 7 to 12 days following cancer chemotherapy. ${ }^{16}$ We defined neutropenia as a neutrophil count $<1.0 \times 10^{7}$.

\section{Data Collection}

A total of 389 patients were triaged as category 2 "hot and high risk" in the study period, of which 244 patients met the inclusion criteria. Data were extracted from the hospital electronic medical record FirstNet (Cerner, Kansas City, Missouri, USA) and the supplementary paper-based medical record. Data collected included patient demographics, emergency and hospital length of stay, in-hospital mortality and number of intensive care admissions. Quantitative data such as time to interventions including antibiotics, fluids and antipyretics were also collected, as were the overall leukocyte and neutrophil counts and any positive culture or imaging results (Figure 1).

\section{Study Outcomes}

The primary outcome for the study was the time to first antibiotics for all patients who met inclusion criteria. Secondary outcomes included time to commencement of the first litre of IV fluids, time to antipyretics which was defined as a dose of paracetamol and/or non-steroidal antiinflammatories and the appropriate choice of first-line antibiotics. Both of these outcomes examined the timings before a definitive diagnosis of febrile neutropenia had been reached and was based solely on the "at risk" triage

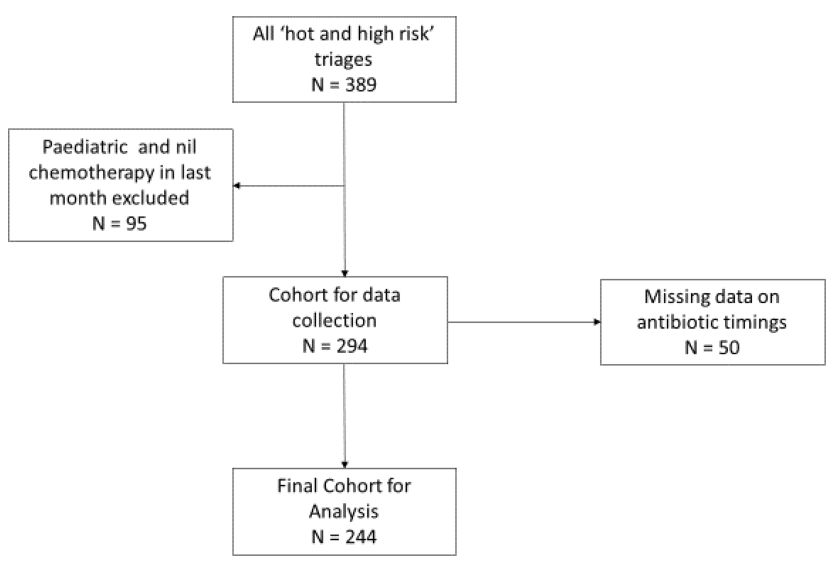

Figure I Cohort inclusion criteria. 
classification of "hot and high risk". Finally, we retrospectively examined if there were differences between the time to care of those found to be subsequently neutropenic.

\section{Statistical Analysis}

Data were collected and collated in Excel 2013 (Microsoft, Redmond, Washington, USA) and analysed using SPSS version 19 (IBM, Armonk, New York, USA). Time to antibiotics, antipyretics and first litre of IV fluids were calculated electronically from initial triage time stamps to time written on the medication or fluid charts for the administration of therapy. ED and hospital length of stay were calculated via electronic triage time stamps and electronic discharge time stamps. Results for primary and secondary outcomes were reported in percentages or means (median; Interquartile range).

\section{Results}

Of the 244 patients, 119 were male and 125 were female with a mean age of 62.7 years $(66.5 ; 54-73$ years) and shown in Table 1 with admission specialty. Presentations were clustered during the summer months of January, February and December. All but two subjects had an initial full blood count taken, with $24.18 \%$ being classed as neutropenic $(\mathrm{N}=59)$. Comparison between the two cohorts of patients (neutropenic $\mathrm{v}$ non-neutropenic) on triage observations is shown in Table 2 .

Table I Demographics And Admission Specialty

\begin{tabular}{|c|c|}
\hline \multicolumn{2}{|l|}{ Total Cohort $(n=244)$} \\
\hline Gender (M:F) & $119: 125$ \\
\hline Mean age (IQR) & $62.7(54-73)$ \\
\hline Admission specialty $(n)$ & \\
\hline - Acute surgical unit & 2 \\
\hline - Gastroenterology & 2 \\
\hline - Emergency medicine & 5 \\
\hline - Geriatrics & 8 \\
\hline - Gynaecology & 1 \\
\hline - Haematology & 51 \\
\hline - Infectious diseases & 7 \\
\hline - Oncology & 92 \\
\hline - Nephrology & 13 \\
\hline - Palliative care & 2 \\
\hline - Respiratory & 17 \\
\hline - Rheumatology & 3 \\
\hline - Urology & 5 \\
\hline - Died in department & 1 \\
\hline - Not admitted & 35 \\
\hline
\end{tabular}

There were 31 patients $(12.70 \%)$ who had no initial antibiotics. Interestingly, 127 patients (52.05\%) received either Piperacillin-Tazobactam (Tazocin) or cefepime with gentamicin as second line if indicated, which is congruent with the local clinical pathway and international guidelines. This meant that one third of patients were given other variations of antibiotic therapy, including 13 $(5.33 \%)$ who received only gentamicin (Figure 2).

The mean time to administration of antibiotics for the cohort was 127 mins (82; 49.5-149 mins). For neutropenic patients, this was 124 mins $(73 ; 42-116)$ whilst for nonneutropenic patients it was 128 mins (84; 52-162 mins). Initial IV fluid boluses were given to 177 patients (72.54\%), with a mean time to initial fluid administration of 166 mins (117; 64-196 mins). 161 patients (65.98\%) received antipyretics, with a mean time to administration of 160 mins (90; 53-208 mins) (Table 3).

Initial blood cultures were taken in 231 patients (94.67\%), with $28(12.12 \%)$ having a lab-confirmed organism whilst urine culture was collected in 159 patients $(65.16 \%)$ and $31(19.50 \%)$ were culture positive. A small number of patients $(5,2.05 \%)$ had a wound culture collected, with three positive results. Chest x-ray was undertaken in 221 subjects $(90.57 \%)$, with 53 (23.98\%) showing infective change on formal radiological reporting (Table 4).

As shown in Table 5, mean ED length of stay was 10 hrs and 27 mins $(6: 32 ; 04: 00-14: 11)$. The number of patients who went to ICU from ED from this cohort was $2(0.8 \%)$ whilst 1 patient died in the emergency department. Over half of the patients in the study cohort were admitted under a medical oncologist or haematologist (58.6\%) whilst $35(14.3 \%)$ did not require admission to hospital. The remaining patients were admitted under a spread of medical and surgical sub-specialties based on clinical symptoms or investigation results.

\section{Discussion}

This study has demonstrated that there is significant delay of initial treatment in our study cohort. Current international guidelines recommend administration of broad-spectrum intravenous antibiotics within 1 hour of initial presentation ${ }^{8}$ Local guidelines within this institution are for antibiotic administration within 30 mins of arrival of piperacillin-Tazobactam (Tazocin) $4.5 \mathrm{~g}$ or Cefepime $2 \mathrm{~g}$ for penicillin-allergic patients. Patients classed as systemically unwell (with $\mathrm{SpO}_{2}<90 \%$, a systolic blood pressure of $<90$, or with new onset confusion) should also receive Gentamicin at 
Table 2 Triage Observations And Initial Blood Results

\begin{tabular}{|c|c|c|c|c|c|c|c|c|c|}
\hline Cohort $(N=244)$ & & $\begin{array}{l}\text { Temperature } \\
\left({ }^{\circ} \mathrm{C}\right)\end{array}$ & $\begin{array}{l}\text { Heart } \\
\text { Rate } \\
\text { (Beats/ } \\
\text { min) }\end{array}$ & $\begin{array}{l}\text { Systolic } \\
\text { Blood } \\
\text { Pressure } \\
(\mathrm{mmHg})\end{array}$ & $\begin{array}{l}\text { Diastolic } \\
\text { Blood } \\
\text { Pressure } \\
\text { (mmHg) }\end{array}$ & $\begin{array}{l}\text { Respiratory } \\
\text { Rate } \\
\text { (Breaths/ } \\
\text { min) }\end{array}$ & $\begin{array}{l}\mathrm{SpO}_{2} \\
(\%)\end{array}$ & $\begin{array}{l}\text { Neutrophil } \\
\text { Count } \\
\left(\times 10^{7}\right)\end{array}$ & Lactate \\
\hline $\begin{array}{l}\text { Neutropenic } \\
N=59\end{array}$ & $\begin{array}{l}\text { Mean } \\
\text { Median } \\
\text { Max } \\
\text { Min } \\
\text { Ist } \\
\text { Quartile } \\
\text { 3rd } \\
\text { Quartile } \\
\text { Number } \\
\text { Missing }\end{array}$ & $\begin{array}{l}38.1 \\
38.2 \\
39.7 \\
36.0 \\
37.5 \\
38.7 \\
6\end{array}$ & $\begin{array}{l}106 \\
108 \\
147 \\
63 \\
92 \\
119 \\
13\end{array}$ & $\begin{array}{l}128 \\
124 \\
166 \\
102 \\
116 \\
140 \\
9\end{array}$ & $\begin{array}{l}73 \\
71 \\
98 \\
57 \\
67 \\
79 \\
9\end{array}$ & $\begin{array}{l}21 \\
20 \\
79 \\
16 \\
18 \\
20 \\
15\end{array}$ & $\begin{array}{l}97 \\
97 \\
100 \\
92 \\
95 \\
98 \\
10\end{array}$ & $\begin{array}{l}0.3 \\
0.2 \\
0.9 \\
0.0 \\
0.0 \\
0.4 \\
0\end{array}$ & $\begin{array}{l}1.8 \\
1.6 \\
5.6 \\
0.8 \\
1.2 \\
2.2 \\
13\end{array}$ \\
\hline $\begin{array}{l}\text { Non-Neutropenic } \\
N=185\end{array}$ & $\begin{array}{l}\text { Mean } \\
\text { Median } \\
\text { Max } \\
\text { Min } \\
\text { Ist } \\
\text { Quartile } \\
\text { 3rd } \\
\text { Quartile } \\
\text { Number } \\
\text { Missing }\end{array}$ & $\begin{array}{l}38.2 \\
38.3 \\
40.4 \\
35.8 \\
37.7 \\
38.9 \\
4\end{array}$ & $\begin{array}{l}107 \\
108 \\
150 \\
52 \\
97 \\
120 \\
17\end{array}$ & $\begin{array}{l}127 \\
126 \\
197 \\
81 \\
109 \\
143 \\
21\end{array}$ & $\begin{array}{l}74 \\
74 \\
106 \\
41 \\
66 \\
81 \\
21\end{array}$ & $\begin{array}{l}21 \\
20 \\
40 \\
16 \\
18 \\
26 \\
47\end{array}$ & $\begin{array}{l}96 \\
96 \\
100 \\
84 \\
94 \\
98 \\
19\end{array}$ & $\begin{array}{l}7.8 \\
6.1 \\
31.7 \\
1.1 \\
3.7 \\
11.1 \\
2\end{array}$ & $\begin{array}{l}1.9 \\
1.7 \\
12.7 \\
0.6 \\
1.3 \\
2.3 \\
31\end{array}$ \\
\hline
\end{tabular}

$7 \mathrm{mg} / \mathrm{kg}$. For patients receiving prophylactic antibiotics preadmission, local guidelines suggest these are withheld and replaced by IV empirical antibiotics until review by the patient's parent specialty. Granulocyte colony-stimulating factor in this institution is given on a case-by-case basis after discussion with the admitting consultant.

The reasons for the results found are likely multifactorial. Staff workload and lack of staff to increase capacity, combined with access block, exit block and ED overcrowding are the most important and relevant reasons for our outcomes. Furthermore, continuing to adhere to the rigid doctor-first approach has meant that patients often

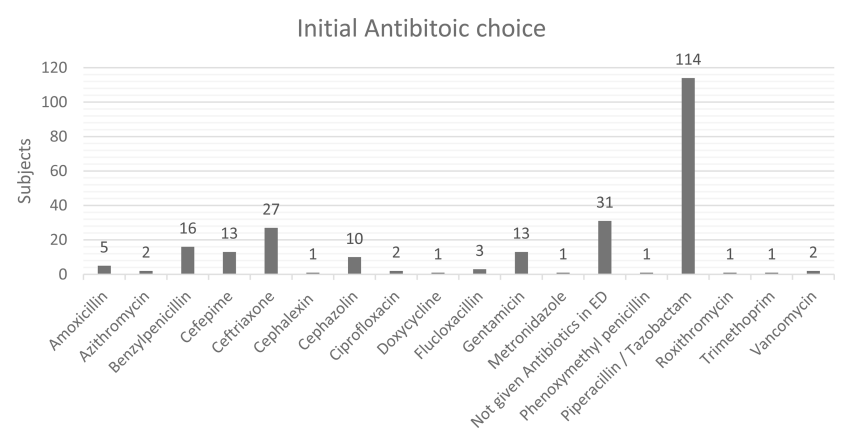

Figure 2 Initial antibiotic selection. wait for medical availability prior to treatment commencement which does not abide by the local guideline of initiation of therapy by nursing staff. Increasing overall patient presentations without significant increases in resource allocation has also reduced the ability to respond to this time-critical condition. Unfortunately, at present, many of these factors are not within the locus of control of the ED staff or management. However, the knowledge of the local guideline and adherence to its recommendations are key to improving care and is controllable. It is not clear whether the delays to antibiotics administration occur in the period between initial triage and review by a clinician or between the time of prescription of antibiotics and their administration given there are no time stamps. With the introduction of electronic prescribing of medications and fluids across New South Wales and Australia, this data will soon be readily available and will assist in identifying the ongoing cause for delays as there will be electronic time stamping of prescription orders. These changes will be key factors for reducing errors and improving the accuracy of data collection for continuing quality improvement whilst improving patient safety and outcomes.

Interestingly in our study, for the neutropenic cohort of $23 \%$, the time to initial IV antibiotics was lower than the 
Table 3 Time To Antibiotics, Fluids And Antipyretics For Cohort Sub-Groups

\begin{tabular}{|l|l|l|l|}
\hline & Mean Time (Minutes) & Median Time (Minutes) & Inter Quartile Range \\
\hline Time To IV Antibiotic & & & $49.5-149$ \\
Total cohort & 127 & 82 & $42-116$ \\
Neutropenic & 124 & 73 & $52-162$ \\
Non-neutropenic & 128 & 84 & \\
\hline Time To Antipyretic & & & $53-208$ \\
Total cohort & 160 & 90 & $48-235$ \\
Neutropenic & 159 & 119 & $56-207$ \\
Non-neutropenic & 160 & 90 & \\
\hline Time To First IV Fluid & & 117 & $64-196$ \\
Total cohort & 166 & 119 & $67-196$ \\
Neutropenic & 176 & 116 & $64-194$ \\
Non-neutropenic & 163 & & \\
\hline
\end{tabular}

Table 4 Number Of Patients With Specific Blood And Urine Culture Positive Samples

\begin{tabular}{|l|l|l|l|}
\hline \multicolumn{2}{|l|}{ Blood Cultures } & Subjects & Urine Cultures \\
\hline Organism & 2 & Organism & Subjects \\
\hline Staph aureus & 2 & E. coli & 20 \\
E. coli & 12 & E. coli and Klebsiella & Enterococcus faecalis \\
MRSA & $\mathrm{I}$ & Proteus mirabilis & 2 \\
Coagulase Negative Staphylococcus & 4 & Pseudomonas aeruginosa & 3 \\
Strep. pneumoniae & 2 & Staph aureus & 2 \\
Fusobacterium species & $\mathrm{I}$ & Stenotrophomonas maltophilia & $\mathrm{I}$ \\
Enterococcus gallinarum & $\mathrm{I}$ & Streptococcus milleri & $\mathrm{I}$ \\
Strep. viridians & 3 & & \\
Pseudomonas aeruginosa & $\mathrm{I}$ & & \\
Strep. viridians/Rhotia & $\mathrm{I}$ & \\
\hline
\end{tabular}

non-neutropenic patients. However, they had a delay to the first litre of IV fluids and antipyretics therapy. This finding is difficult to explain given that both treatments should be given simultaneously and the disparity would suggest that there were other factors involved in how and why the patients received the care that they did which are difficult to identify in a retrospective study but could include increased suspicion of neutropenia given history and time since last chemotherapy, prioritising antibiotics over other therapeutics and some instances of nurse initiation as per the local guideline which does not include other therapeutic interventions.

Another interesting outcome was the number of positive chest x-ray imaging results in our cohort, which is higher than what other studies have demonstrated. The likely causes for this discrepancy include ongoing high burden of chronic disease in western Sydney, especially smoking and environment related obstructive airway disease, which may increase the risk of chest $\mathrm{x}$-ray changes being present. There may also be some degree of existing changes on the imaging reports as we only accessed the report for the current presentation.

In the study, antibiotic selection encompass only the initial antibiotic decision choice prior to a definitive identification of a source of infection or the return of culture results. In some of the cases, deviation from guidelines may have been indicated but would have been dependent upon the clinical situation and judgement of the treating or admitting clinician. However, it is noted that the clinical reasoning for such decisions was not adequately documented in either the electronic or paper records for any patient not prescribed guideline treatment and so this can only remain a consideration until documentation is improved by clinical staff. What we did notice, however, was a tendency for a supervising consultant to prefer certain 
Table 5 Emergency And Hospital Length Of Stay With Cohort Sub-Groups

\begin{tabular}{|l|l|l|}
\hline & $\begin{array}{l}\text { ED Length Of } \\
\text { Stay (d:hh:mm) }\end{array}$ & $\begin{array}{l}\text { Hospital Length } \\
\text { Of stay (d:hh:mm) }\end{array}$ \\
\hline $\begin{array}{l}\text { Total Cohort } \\
\text { Median }\end{array}$ & \\
Mean & $0: 6: 32$ & $3: 3: 47$ \\
IQ Range & $0: 10: 27$ & $7: 15: 49$ \\
\hline Neutropenic Cohort & $00: 04: 00-0: 14: 11$ & $1: 3: 26-5: 20: 22$ \\
Median & $0: 7: 30$ & \\
Mean & $0: 8: 39$ & $5: 2: 05$ \\
IQ Range & $0: 4: 29-0: 12: 08$ & $9: 8: 24$ \\
\hline Non-Neutropenic & & $3: 1: 45-8: 2: 28$ \\
Cohort & & \\
Median & $0: 6: 20$ & $2: 19: 58$ \\
Mean & $0: 11: 01$ & $7: 2: 53$ \\
IQ Range & $0: 3: 57-0: 15: 31$ & $0: 16: 13-5: 5: 37$ \\
\hline
\end{tabular}

antibiotics, regardless of them being emergency or medical consultants. We also found that there was a distinct lack of knowledge of the treatment guidelines and antibiotic selection associated with these amongst the junior staff and this led to increasing utilisation of familiar drugs such as ceftriaxone and benzyl-penicillin instead of the mandated antibiotics within the guidelines.

The study only looked at the use and choice of the initial dose of empirical antibiotics after arrival in the emergency department. It would be interesting to review whether this changed after the specialty team review.

\section{Limitations}

This study was limited given that it was a retrospective chart review. There were noted limitations including missing data points which have likely negatively impacted primary and secondary outcomes in terms of time to initial treatment modalities. We have also not been able to determine the clinical reasoning of decisions to deviate from guideline antibiotic selection due to unclear or insufficient documentation in the medical note. In terms of the increased radiological findings, the study only used presentation imaging results and did not examine previous findings, leading to a possibility of overcalling of current findings which may have already been present on previous imaging.

\section{Conclusion}

This study has shown that there is poor compliance to the local febrile neutropenia guideline and internationally established treatment guidelines with respect to the initial assessment and treatment of patients presenting with "hot and high risk" and having had chemotherapy within 1 month of presentation. Given healthcare systems worldwide are facing increasing demands on emergency care in conjunction with increasing patient presentations and resultant access and exit block, we suggest minimising and streamlining the steps between triage and antibiotic administration for these high-risk patients after identification. In order to address these factors, we suggest the following recommendations:

1. Initiation of preliminary investigations and first dose of intravenous antibiotics at triage, as per local guidelines and protocols, by triage nursing or rapid response medical staff.

2. Staff education on local protocols, and to prompt timely initiation of management in these patients.

3. The utilisation of electronic medical systems to prompt early pathology collection and suggesting antibiotic selection based on guidelines and known allergy status as a pop-up screen or part of a powerorder system which pairs pathology and imaging requests with suggested antibiotic, fluid and antipyretic therapy.

4. Improvements in staff utilisation and increased staffing and resources to match the increasing presentation numbers and access/exit block. We also believe that by alleviating ED overcrowding, treatment initiation for our cohort can conform to local guidelines.

\section{Acknowledgments}

The study was approved by the APOLLO Committee, a subcommittee for quality improvement, as part of the NBMLHD Human Research and Ethics Committee. The approval number for the study is NBM18/745 - 18/29(A). The funding was provided by the Department of Emergency Medicine, Nepean Hospital, Kingswood, NSW, Australia.

\section{Disclosure}

The authors report no conflicts of interest in this work.

\section{References}

1. Kuderer NM, Dale DC, Crawford J, Cosler LE, Lyman GH. Mortality, morbidity and cost associated with febrile neutropenia in adult cancer patients. Cancer. 2006;106:2258-2266. doi:10.1002/(ISSN)1097-0142 
2. Gustinetti G, Mikulska M. Bloodstream infections in neutropenic cancer patients: a practical update. Virulence. 2016;7(3):280-297. doi:10.1080/21505594.2016.1156821

3. Dellinger RP, Levy MM, Rhodes A, et al. Surviving sepsis campaign: international guidelines for management of severe sepsis and septic shock. Intensive Care Med. 2013;39:165-228.

4. Lingaratnam S, Slavin MA, Koczwara B, et al. Introduction to the Australian consensus guidelines for the management of neutropenic fever in adult cancer patients, 2010/2011. Intern Med J. 2011;41:7581. doi:10.1111/j.1445-5994.2010.02338.x

5. Tam CS, O'Reilly M, Andersen D, et al. Use of empiric antimicrobial therapy in neutropenic fever. Intern Med J. 2011;41:90-101. doi:10.1111/j.1445-5994.2010.02340.x

6. Lingaratnam S, Slavin MA, Mileshkin L, et al. An Australian survey of clinical practices in management of neutropenic fever in adult cancer patients 2009. Intern Med J. 2011;41:110-120. doi:10.1111/ j.1445-5994.2010.02342.x

7. Kumar A, Roberts D, Wood KE, et al. Duration of hypotension before initiation of effective antimicrobial therapy is the critical determinant of survival in human septic shock. Crit Care Med. 2006;34(6):1589-1596. doi:10.1097/01.CCM.0000217961.75225. E9

8. Perron T, Emara M, Ahmed S. Time to antibiotics and outcomes in cancer patients with febrile neutropenia. BMC Health Serv Res. 2014;14:162. doi:10.1186/1472-6963-14-162

9. Rhodes A, Evans LE, Alhazzani W, et al. Surviving sepsis Campaign: international guidelines for sepsis management and septic shock 2016. Crit Care Med. 2017;45(3):486-552. doi:10.1097/CCM.0000 000000002255
10. Penack O, Becker C, Buchheidt D, et al. Management of sepsis in neutropenic patients: 2014 updated guidelines from the infectious diseases working party of the German Society of Hematology and Medical Oncology (AGIHO). Ann Hematol. 2014;93(7):1083-1095. doi:10.1007/s00277-014-2086-0

11. Taplitz RA, Kennedy EB, Bow EJ, et al. Outpatient management of fever and neutropenia in adults treated for malignancy: American society of clinical oncology and infectious diseases society of America clinical practice guideline update. J Clin Oncol. 2018;36 (14):1443-1453. doi:10.1200/JCO.2017.77.6211

12. Lingaratnam S, Thursky KA, Slavin MA, Kirsa SW, Bennett CA, Worth LJ. The disease and economic burden of neutropenic fever in adult patients in Australian cancer treatment centres 2008: analysis of the victorian admitted episodes dataset. Intern Med J. 2011;41:121129. doi:10.1111/j.1445-5994.2010.02343.x

13. Neutropenia and risk of infection. CDC Available from: www.cdc.gov/ cancer/preventinfections/pdf/neutropenia.pdf. Accessed August 2, 2017.

14. Mackway-Jones K, Marsden J, Windle J, Editors. Emergency Triage: Manchester Triage Group. 3rd ed. Manchester: Wiley Blackwell; Dec, 2013.

15. Farrohknia N, Castrén M, Ehrenberg A, et al. Emergency department triage scales and their components: a systematic review of the scientific evidence. Scand J Trauma Resusc Emerg Med. 2011;19:42. doi:10.1186/1757-7241-19-42

16. Worth LJ, Lingaratnam S, Taylor A, et al., Australian Consensus Guidelines 2011 Steering Committee. Use of risk stratification to guide ambulatory management of neutropenic fever. Australian consensus guidelines 2011 steering committee. Intern Med J. 2011;41 (1b):82-89. doi:10.1111/j.1445-5994.2010.02339.x
Clinical Audit

\section{Publish your work in this journal}

Clinical Audit is an international, peer-reviewed, open access journal focusing on the processes and outcomes of clinical audit in any area of healthcare. All aspects of patient care are addressed within the journal and practitioners from all disciplines are invited to submit their work. Areas covered include: Publication of audits; How an audit has changed practice; Practical tips on how to do audits and to

Submit your manuscript here: https://www.dovepress.com/clinical-audit-journal avoid pitfalls; How audits have changed patient care; Calls and justifications for new audits. The manuscript management system is completely online and includes a very quick and fair peer-review system, which is all easy to use. Visit http://www.dovepress.com/ testimonials.php to read real quotes from published authors. 